\title{
A novel putative auxin carrier family regulates intracellular auxin homeostasis in plants
}

Elke Barbez ${ }^{1,2}$, Martin Kubeš ${ }^{3}$, Jakub Rolčík ${ }^{4}$, Chloé Béziat ${ }^{1,2}$, Aleš Pěnčík ${ }^{5}$, Bangjun Wang ${ }^{6}$, Michel Ruiz Rosquete ${ }^{1,2}$, Jinsheng Zhu ${ }^{6}$, Petre I. Dobrev ${ }^{3}$, Yuree Lee ${ }^{7}$, Eva Zažímalovà ${ }^{3}$, Jan Petrášek ${ }^{3}$, Markus Geisler ${ }^{6}$, Jiří Friml I Jürgen Kleine-Vehn $^{1,2}$

The phytohormone auxin acts as a prominent signal, providing, by its local accumulation or depletion in selected cells, a spatial and temporal reference for changes in the developmental program ${ }^{1-7}$. The distribution of auxin depends on both auxin metabolism (biosynthesis, conjugation and degradation $)^{8-10}$ and cellular auxin transport ${ }^{11-15}$. We identified in silico a novel putative auxin transport facilitator family, called PIN-LIKES (PILS). Here we illustrate that PILS proteins are required for auxin-dependent regulation of plant growth by determining the cellular sensitivity to auxin. PILS proteins regulate intracellular auxin accumulation at the endoplasmic reticulum and thus auxin availability for nuclear auxin signalling. PILS activity affects the level of endogenous auxin indole-3-acetic acid (IAA), presumably via intracellular accumulation and metabolism. Our findings reveal that the transport machinery to compartmentalize auxin within the cell is of an unexpected molecular complexity and demonstrate this compartmentalization to be functionally important for a number of developmental processes.

Prominent auxin carriers with fundamental importance during plant development are PIN-FORMED (PIN) proteins ${ }^{1-3,6,9,15}$. PIN1type auxin carriers regulate the directional intercellular auxin transport at the plasma membrane. In contrast, atypical family member PIN5 regulates intracellular auxin compartmentalization into the lumen of the endoplasmic reticulum and its role in auxin homeostasis was recently identified $^{15,16}$. PIN proteins have a predicted central hydrophilic loop, flanked at each side by five transmembrane domains. We screened in silico for novel PIN-like putative carrier proteins with a predicted topology similar to PIN proteins (Fig. 1a and Supplementary Fig. 2) and identified a protein family of seven members (Fig. 1b) in Arabidopsis thaliana, which we designated as the PILS proteins. In contrast to the similarities in the predicted protein topology, PIN and PILS proteins do not show pronounced protein sequence identity (10-18\%), which limits the identification of PILS proteins by conventional, reciprocal basic local alignment search tool (BLAST) approaches. However, the distinct PIN and PILS protein families contain both the InterPro auxin carrier domain which is an insilico-defined domain, aiming to predict auxin transport function (http://www.ebi.ac.uk/panda/InterPro.html). The PILS putative carrier family is conserved throughout the whole plant lineage, including unicellular algae (such as Ostreococcus tauri and Chlamydomonas reinhardtii) (Supplementary Fig. 3) where PIN proteins are absent ${ }^{16}$, indicating that PILS proteins are evolutionarily older.

PILS genes are broadly expressed in various tissues (Fig. 1c) and PILS2-PILS7 were transcriptionally upregulated by auxin application in wild-type seedlings (Fig. 1d-f and Supplementary Fig. 4), indicating a role in auxin-dependent processes. To investigate the potential function of the putative PILS auxin flux facilitators in plant development, we overexpressed PILS proteins using the constitutive, viral $35 S$ promoter. Ectopic expression of PILS genes, such as PILS1 or PILS3, resulted in dwarfed and/or bushy plants showing severe defects in flower development, leading to sterility in the T1 generation (Fig. 2a-d). Flowers of these PILS-overexpressing plants showed severe patterning defects, such as homeotic transformation of flower organs into new flower buds, triplication of the gynoecium or unfused carpels (Fig. 2b-d). To circumvent sterility, we screened for weaker p35S::PILS lines and isolated moderately PILS5 overexpressing lines showing fertile flower development.

To assess further the developmental importance and potential redundancy of PILS proteins in auxin-regulated processes, we focused
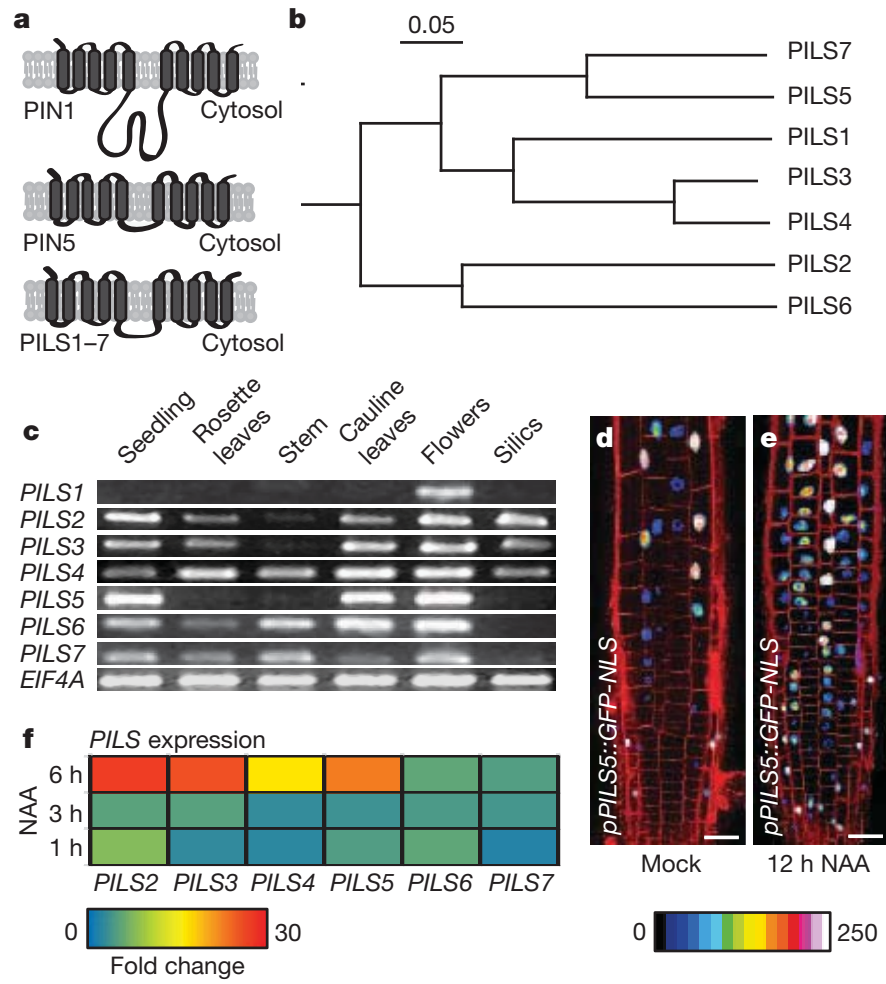

Figure $1 \mid$ Novel PILS protein family. a, Predicted topology of both PIN1-/ PIN5-type PIN subfamilies and PILS proteins. b, Phylogenetic tree of the $A$. thaliana PILS proteins. Scale depicts 0.05 substitutions per position. $\mathbf{c}$, Reverse transcription PCR (RT-PCR) of the seven PILS genes and EIF4A (control) in several plant tissues. d, e, 1-Naphthaleneacetic acid (NAA; $10 \mu \mathrm{M}, 12 \mathrm{~h}$ )induced $p$ PILS5::GFP-NLS (GFP fused to nuclear localisation signal, NLS) expression in the root transition zone. Colour-code (black to white) depicts (low to high) GFP signal intensity. Propidium-iodide-stained cell walls in red. Scale bar, $25 \mu \mathrm{m}$. f, Quantitative RT-PCR of PILS2-7 after $10 \mu \mathrm{M}$ NAA treatment for 1, 3 and $6 \mathrm{~h}$. Colour code from blue (low) to red (high) depicts fold changes (0-30) (see also Supplementary Fig. 4).

${ }^{1}$ Department of Plant Systems Biology, VIB and Department of Plant Biotechnology and Bioinformatics, Ghent University, 9052 Gent, Belgium. ${ }^{2}$ Department of Applied Genetics and Cell Biology, University of Natural Resources and Life Siences (BOKU), 1190 Vienna, Austria. ${ }^{3}$ Institute of Experimental Botany, The Academy of Sciences of the Czech Republic, 16502 Praha 6, Czech Republic. ${ }^{4}$ Laboratory of Growth Regulators, Faculty of Science, Palacký University and Institute of Experimental Botany AS CR, Šlechtitelů 11,78371 Olomouc, Czech Republic. ${ }^{5}$ Centre of the Region Haná for Biotechnological and Agricultural Research, Department of Growth Regulators, Faculty of Science, Palacký University, Šlechtitelů 11, 78371 Olomouc, Czech Republic. ${ }^{6}$ Department of Biology - Plant Biology, University of Fribourg, Chemin du Musée 10, 1700 Fribourg, Switzerland. ${ }^{7}$ Department of Plant Molecular Biology, University of Lausanne, Quartier Sorge, 1015 Lausanne, Switzerland. 

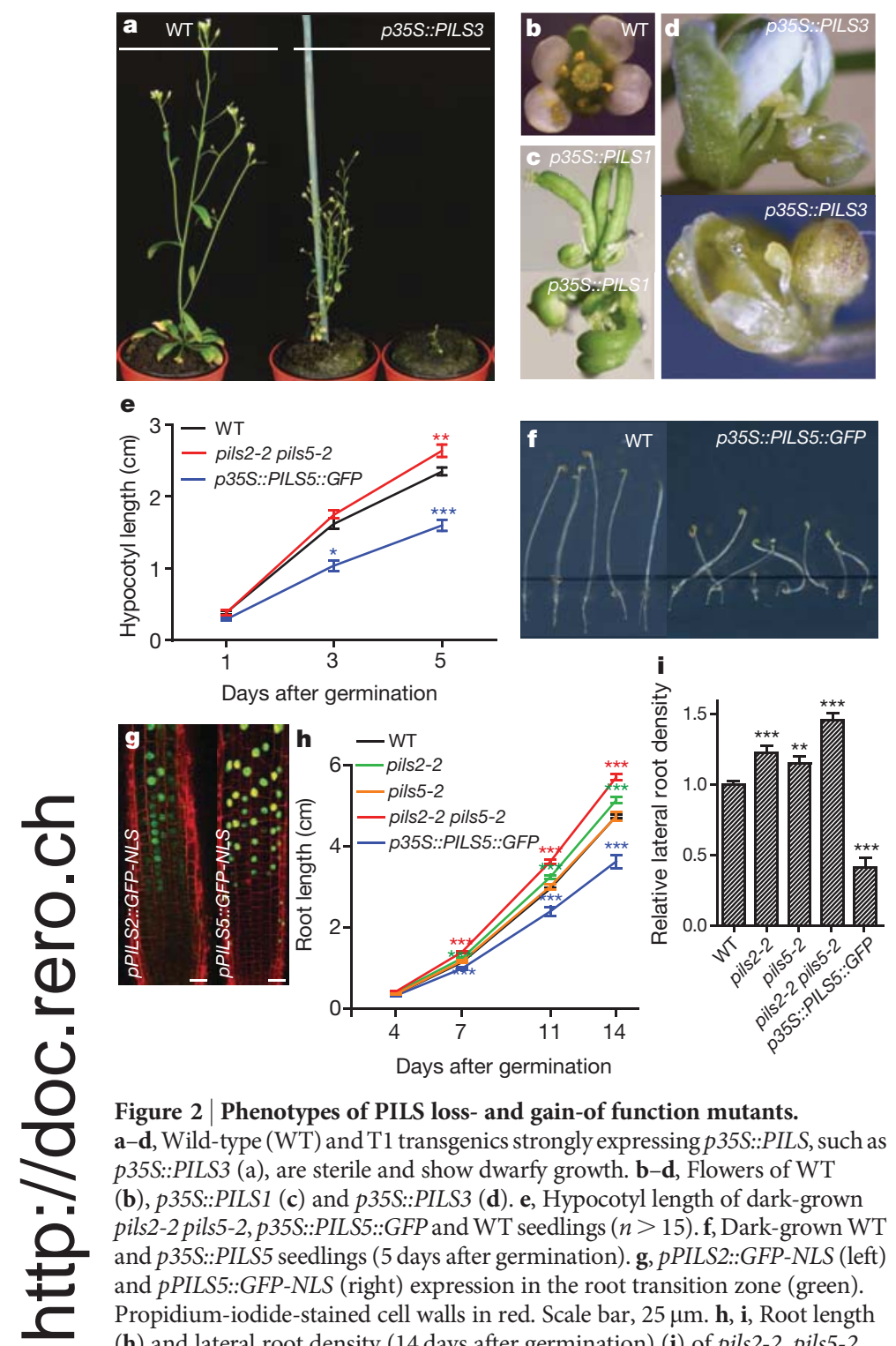

Figure $2 \mid$ Phenotypes of PILS loss- and gain-of function mutants.

a-d, Wild-type (WT) and T1 transgenics strongly expressing $p 35 S:: P I L S$, such as p35S::PILS3 (a), are sterile and show dwarfy growth. b-d, Flowers of WT (b), p35S::PILS1 (c) and p35S::PILS3 (d). e, Hypocotyl length of dark-grown pils2-2 pils5-2, p35S::PILS5::GFP and WT seedlings $(n>15)$. f, Dark-grown WT and $p 35 S:: P I L S 5$ seedlings (5 days after germination). g, pPILS2::GFP-NLS (left) and PPILS5::GFP-NLS (right) expression in the root transition zone (green). Propidium-iodide-stained cell walls in red. Scale bar, $25 \mu \mathrm{m}$. h, i, Root length (h) and lateral root density (14 days after germination) (i) of pils2-2, pils5-2, pils2-2 pils5-2, p35S::PILS5::GFP and WT seedlings $(n>40)$. Error bars represent s.e.m. Student $t$-test $P$-values: ${ }^{*} P<0.05,{ }^{*} P<0.001,{ }^{* * *} P<0.0001$.

on PILS2 and PILS5, because they are the most abundantly expressed PILS genes in seedlings (Fig. 1c) and display partially overlapping expression domains (Fig. 2g). Initially, we investigated PILS function in auxin-dependent hypocotyl and root growth. Dark-grown p35S::PILS5::GFP-expressing (coding for a PILS5-green fluorescent protein (GFP) fusion) and pils2 pils5 double-mutant seedlings showed reduced and enhanced hypocotyl growth, respectively (Fig. 2e and Supplementary Fig. 5). PILS5 gain-of-function also resulted in agravitropic hypocotyl growth (Fig. 2f). PILS2 and PILS5 showed a particular overlapping expression in the root transition zone (Fig. 2g), suggesting a redundant role in regulating root growth. Indeed, pils 2 single-mutant and more pronounced pils 2 pils 5 doublemutant seedlings showed significantly longer roots compared to wildtype seedlings, whereas seedlings overexpressing PILS5 had shorter roots (Fig. 2h). Collectively, our data indicate the requirement of defined PILS protein activity for auxin-dependent growth regulation.

Beside the root and shoot organ growth, auxin tightly controls de novo organ formation such as lateral root organogenesis ${ }^{1}$. Intriguingly, pils2 and pils5 single-mutants and more pronounced pils2 pils5 double-mutants showed higher lateral root density (Fig. 2i). On the contrary, PILS5 gain-of-function reduced lateral rooting (Fig. 2i).
These findings indicate developmental importance of PILS proteins in auxin-regulated processes, such as de novo organ formation and growth regulation.

Next we investigated whether auxin responses are affected in pils mutants using the auxin response reporter DR5 (ref. 17). pils2-2 knockdown and pils5-2 knockout mutants did not show altered DR5 activity in the main root tips (Supplementary Fig. 6a), but showed higher pDR5rev::GFP signal intensity in lateral roots (Fig. 3a). Moderately $p 35 S:: P I L S 5:: G F P$-expressing seedlings showed a visibly reduced auxin response maximum in the very root tip of main (Fig. 3b) and lateral roots (Supplementary Fig. 6b). To distinguish between direct and indirect effects, we investigated the effect of PILS proteins on nuclear auxin signalling at the cellular level. Therefore, we transiently co-expressed PILS2 or PILS5 together with the auxin response reporter $p D R 5 r e v:: m R F P e r$ (expressing the red fluorescent protein (RFP) in response to auxin signalling) in tobacco Bright Yellow 2 (BY-2) cells. PILS2 or PILS5 expression in BY-2 cells reduced auxin signalling as visualized by $p D R 5$ rev::mRFPer activity (Fig. 3c, d). These findings indicate that PILS proteins negatively affect nuclear auxin signalling, presumably by affecting cellular auxin homeostasis.

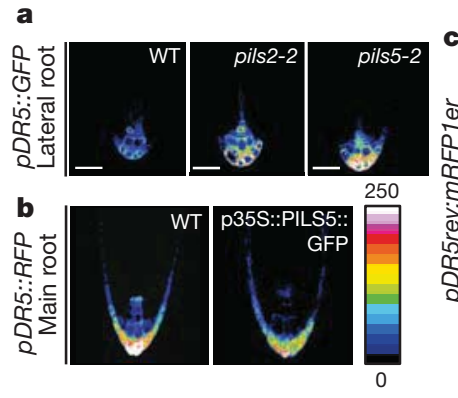

e
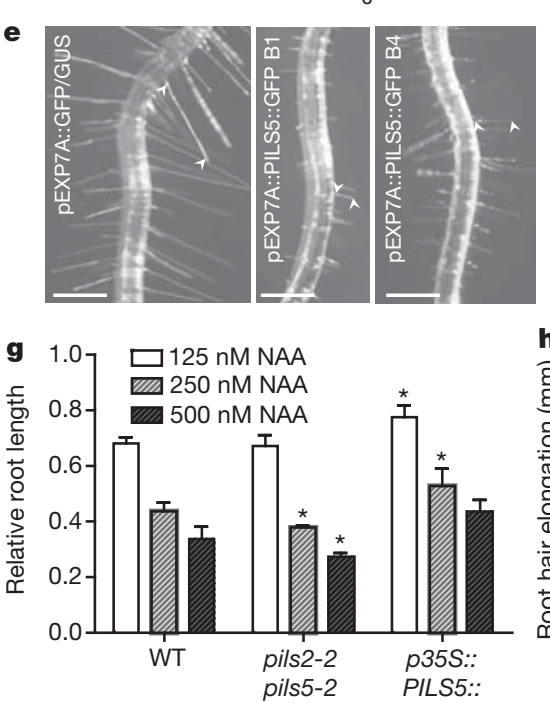

GFP
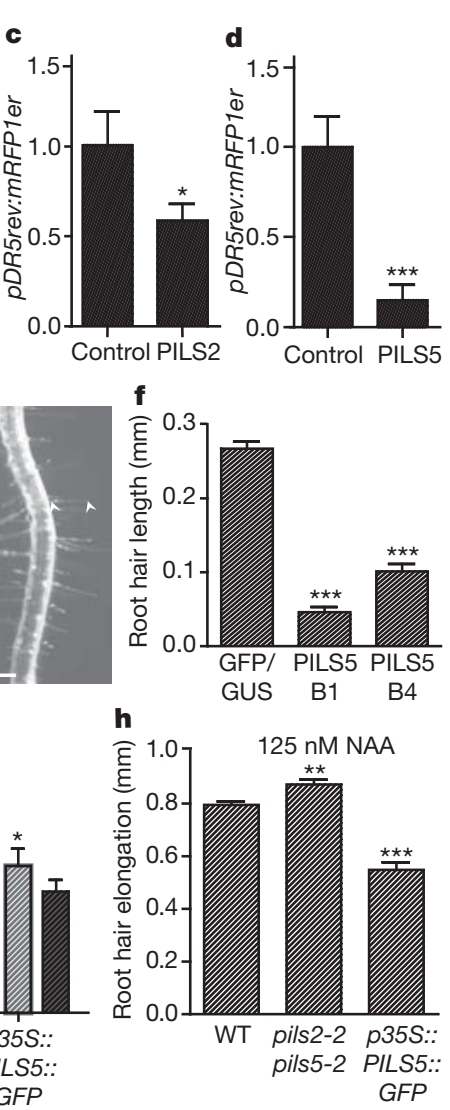

Figure $3 \mid$ PILS proteins affect auxin-dependent cellular growth. a, b, DR5 promoter activity in pils2-2 and pils5-2 (lateral roots) (a) and p35S::PILS5::GFP (main root) (b) compared to wild type (WT) (see also Supplementary Fig. 6). Colour-code (black to white) depicts (low to high) pDR5rev::GFP and pDR5rev::mRFP1er signal intensity. Scale bars, $25 \mu \mathrm{m}$. c, d, Mean grey value of pDR5rev::mRFP1er signal intensity in BY-2 cells expressing p35S::PILS2 (c) or p35S::PILS5 (d) compared to control cells ( $p 35 S:: G F P:: H D E L)$ ( $n>60$ cells). e, f, Root hair length (e, arrowheads) and quantification (f) of transgenic lines expressing $p E X P 7 A:: G F P:: G U S$ (control) and $p E X P 7 A:: P I L S 5: G F P$ (independent lines B1 and B4) $(n=20$ seedlings with 400 counted root hairs in total). Scale bars, $250 \mu \mathrm{m}$. g, Relative root length of NAA-treated pils2-2 pils5-2 and p35S::PILS5::GFP seedlings compared to WT $(n>20)$. $\mathbf{h}$, Auxin-induced root hair elongation ( $n=20$ seedlings with 400 counted root hairs in total). Mean untreated root hair length was subtracted from the treated values. Error bars represent s.e.m. Student $t$-test $P$-values: ${ }^{*} P<0.05,{ }^{* *} P<0.001,{ }^{* * *} P<0.0001$. 
To address whether cellular PILS action on auxin signalling affects cellular growth, we expressed PILS1, PILS3 and PILS5 under a roothair-specific promoter. Deviations in free (active) indole-3-acetic acid (IAA) levels or in auxin signalling induce (high levels) or repress (low levels) root hair growth ${ }^{18}$. As expected, the root-hair-specific expression of PILS1, PILS3 and PILS5 significantly reduced root hair length (Fig. 3e, fand Supplementary Fig. 7a-c), possibly due to PILS-dependent regulation of auxin homeostasis and signalling.

Next we tested whether PILS proteins affect auxin-dependent cellular growth responses. We treated PILS gain- and loss-of-function mutants with auxin that inhibits primary root growth. pils2 pils5 lossand PILS5 gain-of-function mutants showed hyper- and hyposensitive root growth, respectively (Fig. $3 \mathrm{~g}$ ). In agreement with these observations, the auxin-promoted root hair growth was also enhanced in pils2 pils5 double-mutants and reduced in PILS5 overexpressers (Fig. 3h). This set of data indicates that PILS putative auxin facilitators modulate auxin-dependent growth responses during plant development.

To unravel the mechanism by which PILS proteins regulate auxindependent plant development, we investigated the subcellular localization of PILS proteins. We introduced amino- or carboxy-terminal GREEN/RED FLUORESCENT PROTEIN (GFP/RFP) fusions with PILS proteins and transiently or stably expressed these fusion proteins in tobacco BY-2 cell culture, Arabidopsis seedlings and heterologously in yeast (Fig. 4a-c and Supplementary Fig. 8). PILS1, PILS2, PILS3, PILS5, PILS6 and PILS7 GFP/RFP fusion proteins localized to the endoplasmic reticulum in all analysed systems (Fig. $4 \mathrm{a}-\mathrm{c}$ and Supplementary Figs 8 and 10c). In contrast, N- and C-terminal PILS4 fusions did not show detectable fluorescence. The transgenic pPILS5::PILS5::GFP also showed endoplasmic reticulum localization (Fig. 4a) and complemented the pils2 pils5 double-mutant to the pils2 single-mutant level (Supplementary Fig. 9), indicating PILS function at the endoplasmic reticulum.

Next, we addressed whether putative PILS auxin carriers affect cellular auxin accumulation. We generated PILS2 oestradiol-inducible tobacco BY-2 cell cultures and performed ${ }^{3} \mathrm{H}-\mathrm{IAA}$ accumulation assays. PILS2 induction increased the accumulation of radioactivity in BY-2 cells (Fig. 4d). In accordance with the auxin accumulation assays in BY-2 cells, pils2 pils5 double-mutant protoplasts showed significantly higher auxin export (Fig. 4e and Supplementary Fig. 10a), indicating reduced auxin retention capacity in pils 2 pils5 loss-offunction mutants. These gain and loss-of-function studies consistently illustrate that PILS2 and PILS5 function at the endoplasmic reticulum controls cellular accumulation of auxin.

To additionally address PILS protein function in a non-plant system, we expressed PILS3, PILS5 and PILS7 (tagged to GFP or haemagglutinin (HA)) in Saccharomyces cerevisiae yeast cells. In accordance with the effect of PILS2 and PILS5 in plant cells, the expression of A. thaliana PILS3, PILS5 and PILS7 in yeast also increased retention of exogenously applied auxin (Fig. 4f and Supplementary Fig. 10b). To assess the specificity to auxin we used the common organic control benzoic acid (BA). Notably, PILS3, PILS5 and PILS7 did not affect the cellular accumulation of BA (Fig. $4 \mathrm{f}$ and Supplementary Fig. 10b). These findings indicate that PILS proteins specifically regulate cellular auxin accumulation.

PILS proteins increase cellular auxin accumulation, but decrease auxin signalling, which is reminiscent of the regulation of auxin metabolism shown for the PIN5 auxin transporter ${ }^{16}$. To assess the potential action of PILS proteins on auxin metabolism, we analysed the effect of PILS proteins on the auxin metabolism using the inducible PILS2 BY-2 cell line. The high-performance liquid chromatography (HPLC) chromatogram of IAA showed a PILS2-dependent reduction of free IAA within $20 \mathrm{~min}$ (Fig. $4 \mathrm{~g}, \mathrm{~h}$ ), indicating that PILS2 activity affects auxin metabolism.

In accordance with the auxin metabolite profiling in BY-2 cell cultures, pils2, pils5 and pils2 pils5 mutant Arabidopsis seedlings showed significantly higher free IAA levels compared to wild-type seedlings (Fig. 4i). Intriguingly, the ratio of amid auxin conjugates IAA-Glutamate (Glu)
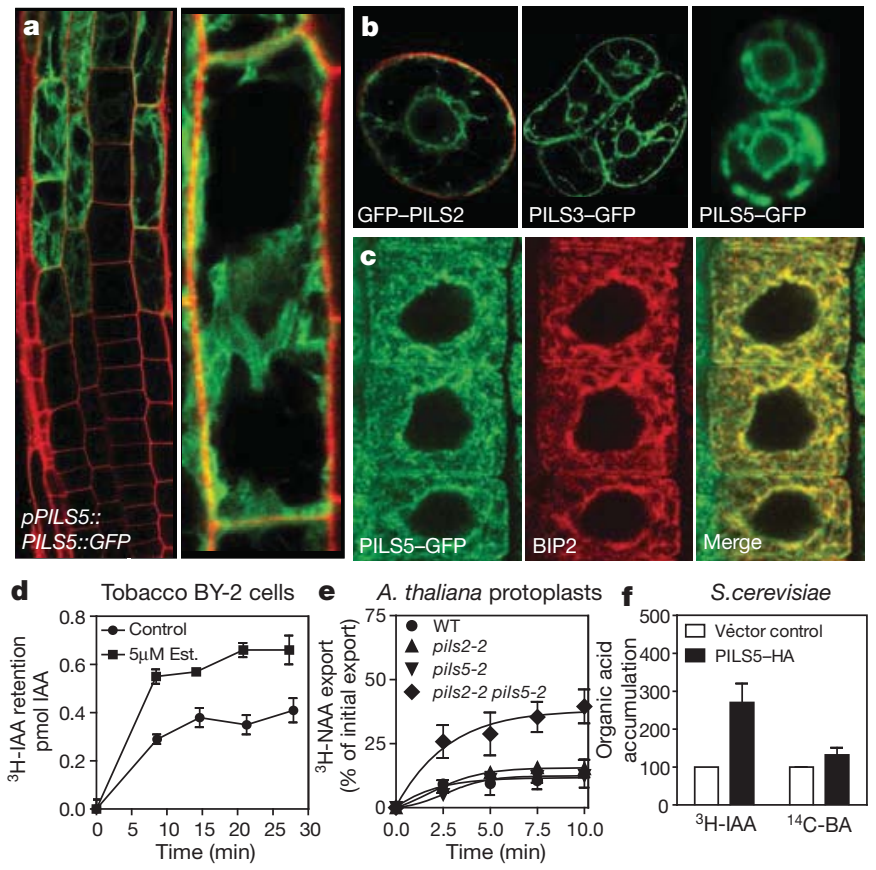

A. thaliana protoplasts $\mathbf{f}$ S.cerevisiae

g Metabolic profile in control cells $\mathbf{h}$ Metabolic profile in PILS2-induced cells
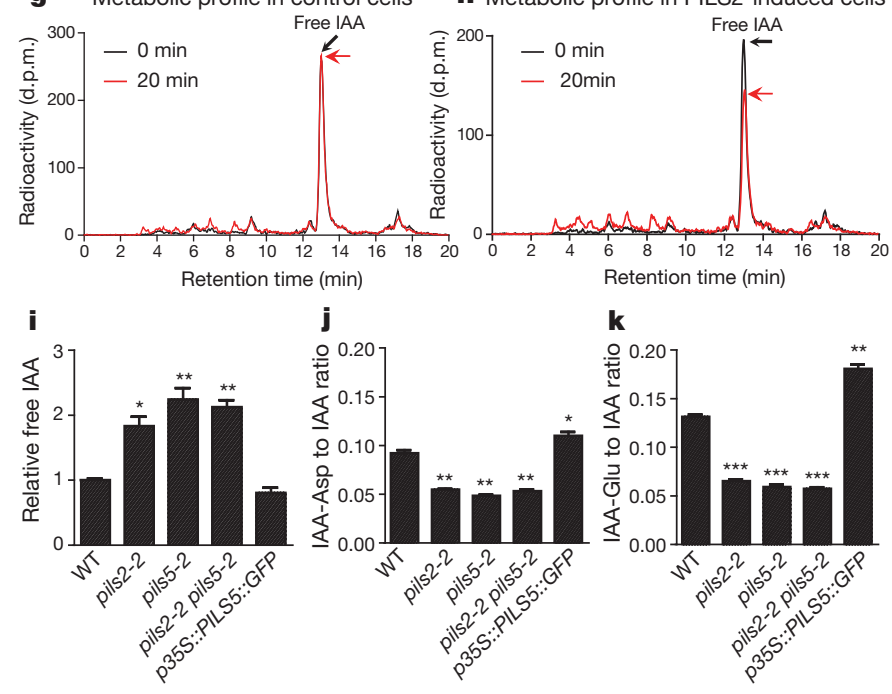

$\mathbf{k}$

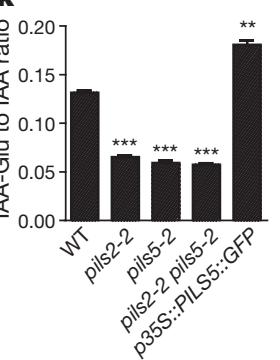

Figure $4 \mid$ PILS involvement in cellular auxin homeostasis. a-c, GFP-PILS2 (b), PILS3-GFP (b), PILS5-GFP (a-c) under endogenous (a) or constitutive promoter $(\mathbf{b}, \mathbf{c})$ in $A$. thaliana root $(\mathbf{a}, \mathbf{c})$, tobacco BY-2 cells (b, left panels) and Saccharomyces cerevisiae yeast cells (b, right panel). c, Immunocytochemistry of PILS5-GFP and the endoplasmic reticulum marker BIP2. $\mathbf{d},{ }^{3} \mathrm{H}$-IAA retention in tobacco BY-2 cells upon oestradiol (Est.)-induced PILS2 expression ( $n=3$ repetitions). e, ${ }^{3} \mathrm{H}-\mathrm{NAA}$ export assay in $A$. thaliana protoplasts of wild type (WT), pils2-2, pils5-2 and pils2 pils5 mutants ( $n=3$ repetitions; see also Supplementary Fig. 10a). f, Accumulation of auxin and benzoic acid in Saccharomyces cerevisiae yeast cells transformed with $p$ GPD::PILS5::HA or an empty vector ( $n=3$ repetitions; see also Supplementary Fig. $10 \mathrm{~b}) . \mathbf{g}, \mathbf{h},{ }^{3} \mathrm{H}-\mathrm{IAA}$ metabolic profile (HPLC chromatogram) in PILS2 non-oestradiol-induced (g) and oestradiol-induced (h) tobacco BY-2 cells after 0 (black line) and 20 min (red line). i-k, Liquid chromatography-mass spectrometry (LC-MS)-derived free IAA levels (i) and ratios of IAA-Asp (j) and IAA-Glu (k) to free IAA in WT, pils2-2, pils5-2, pils2-2 pils5-2 and p35S::PILS5::GFP ( $n=3$ repetitions). Error bars represent s.e.m. Student $t$-test $\mathrm{P}$-values: ${ }^{*} P<0.05$, $* * P<0.001$, $* * * P<0.0001$. Scale bar, $10 \mu \mathrm{m}$.

and IAA-Aspartate (Asp) to free IAA was also significantly shifted towards free IAA in pils2 and pils5 loss-of-function mutants (Fig. 4j, k). Moderately $p 35$ S::PILS5::GFP-expressing seedlings showed a visibly reduced auxin response maximum in the very root tip (Fig. 3b), but only a mild and statistically non significant reduction in free IAA levels 
at the whole seedling level (Fig. 4i). However, the ratio of IAA-Glu and IAA-Asp to free IAA was significantly shifted towards the conjugates in $p 35 S:: P I L S 5:: G F P$ seedlings (Fig. 4j, k), indicating a higher rate of auxin conjugation.

These findings are indicative of a PILS function in cellular auxin homeostasis by regulating auxin metabolism. Hence, we propose a model in which PILS proteins at the endoplasmic reticulum membrane facilitate intracellular auxin accumulation, which seems to contribute to the possibly compartmentalized regulation of auxin metabolism (Supplementary Fig. 1). It is tempting to speculate that auxin conjugation could also take place in the endoplasmic reticulum, although the molecular components remain to be identified.

Our in silico and reverse genetics approaches led to the identification of a novel family of putative auxin transport facilitators. All our genetic, pharmacological, cell biological, physiological and biochemical approaches consistently suggest that PILS proteins function at the endoplasmic reticulum membrane, regulate intracellular auxin accumulation and affect free IAA levels, presumably through conjugationbased auxin metabolism. The PILS action on cellular auxin homeostasis is reminiscent of the function of the atypical PIN family member PIN5 (ref. 16). We uncovered that an additional, distinct protein family regulates intracellular auxin homeostasis. Our analyses of the PILS proteins suggest that intracellular auxin transport and, hence, auxin compartmentalization might be evolutionarily older than directional, cell-to-cell PIN-dependent auxin transport mechanisms. The identification of a novel protein family for the regulation of intracellular auxin homeostasis highlights the evolutionary and developmental importance of intracellular auxin transport. Further studies will address the potential interplay or possible diversified function of endoplasmic reticulum localized PIN5 and PILS1-7 proteins.

\section{METHODS SUMMARY}

Plant material and growth conditions. We used Arabidopsis thaliana of ecotype Columbia 0 (Col-0). The Nicotiana tabacum L. cv. Bright Yellow 2 (BY-2) cell line $^{19}$ was used as suspension-cultured cells. pils2-1 (SALK_024808), pils2-2 (SALK_125391), pils5-1 (SALK_070653) and pils5-2 (SALK_072996) were obtained from the Nottingham Arabidopsis Stock Centre (NASC). Gateway cloning was used to construct pPILS2::GFP/GUS, pPILS5::GFP/GUS, p35S::PILS1-7, p35S::GFP::PILS1-7, p35S::PILS1-7::GFP, pPILS5::PILS5::GFP and $P M D C 7 \_B(p U B Q):: P I L S 2$. The following lines and constructs have been previously described: $p D R 5$ rev::mRFP1er ${ }^{20}$, pDR5rev::GFP and $p 35 S:: G F P:: H D E L^{21}$. Seeds were stratified at $4^{\circ} \mathrm{C}$ for 2 days in the dark. Seedlings were grown vertically on half Murashige and Skoog medium. Plants were grown under long-day (16 h light/ $8 \mathrm{~h}$ dark) conditions at $20-22^{\circ} \mathrm{C}$.

1. Benková, E. etal. Local, efflux-dependent auxin gradients as a common module for plant organ formation. Cell 115, 591-602 (2003).

2. Friml, J. et al. Efflux-dependent auxin gradients establish the apical-basal axis of Arabidopsis. Nature 426, 147-153 (2003).

3. Reinhardt, D. et al. Regulation of phyllotaxis by polar auxin transport. Nature $\mathbf{4 2 6}$, 255-260 (2003).
4. Leyser, O. Dynamic integration of auxin transport and signalling. Curr. Biol. 16, R424-R433 (2006).

5. Dubrovsky, J. G. et al. Auxin acts as a local morphogenetic trigger to specify lateral root founder cells. Proc. Natl Acad. Sci. USA 105, 8790-8794 (2008).

6. Sorefan, K. et al. A regulated auxin minimum is required for seed dispersal in Arabidopsis. Nature 459, 583-586 (2009).

7. Prasad, K. et al. Arabidopsis PLETHORA transcription factors control phyllotaxis. Curr. Biol. 21, 1123-1128 (2011)

8. Woodward, A. W. \& Bartel, B. Auxin: regulation, action, and interaction. Ann. Bot. 95, 707-735 (2005).

9. Ikeda, Y. et al. Local auxin biosynthesis modulates gradient-directed planar polarity in Arabidopsis. Nature Cell Biol. 11, 731-738 (2009)

10. Zhao, Y. Auxin biosynthesis and its role in plant development. Annu. Rev. Plant Biol. 61, 49-64 (2010).

11. Bennett, M. J. et al. Arabidopsis AUX1 gene: a permease-like regulator of root gravitropism. Science 273, 948-950 (1996).

12. Luschnig, C., Gaxiola, R. A., Grisafi, P. \& Fink, G. R. EIR1, a root-specific protein involved in auxin transport, is required for gravitropism in Arabidopsis thaliana. Genes Dev. 12, 2175-2187 (1998).

13. Geisler, M. et al. Cellular efflux of auxin catalyzed by the Arabidopsis MDR/PGP transporter AtPGP1. Plant J. 44, 179-194 (2005).

14. Petrášek, J. et al. PIN proteins perform a rate-limiting function in cellular auxin efflux. Science 312, 914-918 (2006).

15. Zažímalová, E., Murphy, A. S., Yang, H., Hoyerová, K. \& Hošek, P. Auxin transporterswhy so many? Cold Spring Harb. Perspect. Biol. 2, a001552 (2010).

16. Mravec, J. et al. ER-localized PIN5 auxin transporter mediates subcellular homeostasis of phytohormone auxin. Nature 459, 1136-1140 (2009).

17. Ulmasov, T., Murfett, J., Hagen, G. \& Guilfoyle, T. J. Aux/IAA proteins repress expression of reporter genes containing natural and highly active synthetic auxin response elements. Plant Cell 9, 1963-1971 (1997).

18. Lee, S. H. \& Cho, H. T. PINOID positively regulates auxin efflux in Arabidopsis root hair cells and tobacco cells. Plant Cell 18, 1604-1616 (2006).

19. Nagata, T., Nemoto, Y. \& Hasezawa, S. Tobacco BY-2 cell line as the "HeLa" cells in the cell biology of higher plants. Int. Rev. Cytol. 132, 1-30 (1992).

20. Marin, E. et al. miR390, Arabidopsis TAS3 tasiRNAs, and their AUXIN RESPONSE FACTOR targets define an autoregulatory network quantitatively regulating lateral root growth. Plant Cell 22, 1104-1117 (2010).

21. Langhans, M. et al. In vivo trafficking and localization of p24 proteins in plant cells. Traffic 9, 770-785 (2008).

Acknowledgements We are grateful to C. Braeckman for plant transformation; W. Ardiles for sequencing support; L. Charrier for technical assistance; A. Maizel, N. Geldner and P. Pimpl for providing material; J.K.-V. group members for critical reading of the manuscript and the BOKU-VIBT Imaging Center for access and expertise. This work was supported by the Vienna Science and Technology Fund (WWTF) (to J.K.-V.), the Agency for Innovation by Science and Technology (IWT) (predoctoral fellowship to E.B.), the Odysseus program of the Research Foundation-Flanders (to J.F.), the Swiss National Funds (to M.G.) the Ministry of Education, Youth and Sports of the Czech Republic (LC06034) (to E.Z.), Grant Agency of the Czech Republic project P305/ $11 / 2476$ (to J.P.) and P305/11/0797 (to E.Z).

Author Contributions E.B. and J.K.V. conceived the project. E.B. carried out most of the experiments. M.K., P.I.B., E.Z. and J.P. performed auxin metabolite profile and auxin accumulation in BY-2. C.B. analysed auxin-dependent PILS expression and contributed to phenotype analysis. M.R.R. contributed to PILS cloning. J.R. and A.P. measured auxin content in Arabidopsis. B.W., J.Z. and M.G. performed auxin accumulation in yeast and protoplasts. Y.L. modified the oestradiol-inducible vector. E.B., M.K., J.R., E.Z., J.P., M.G., J.F. and J.K.V. discussed the experimental procedures. All authors analysed and discussed the data; E.B. and J.K.V. wrote the paper and all authors saw and commented on the manuscript. 


\section{METHODS}

PILS gene accession codes. Sequence data from this article can be found in The Arabidopsis Information Resource (TAIR; http://www.arabidopsis.org/) or GenBank/EMBL databases under the following accession numbers: PILS1 (At1g20925), PILS2 (At1g71090), PILS3 (Atlg76520), PILS4 (At1g76530), PILS5 (At2g17500), PILS6 (At5g01990), PILS7 (At5g65980).

Plant material, growth conditions and DNA constructs. We used Arabidopsis thaliana of ecotype Columbia 0 (Col-0). The Nicotiana tabacum L. cv. Bright Yellow 2 (BY-2) cell line ${ }^{19}$ was used as suspension-cultured cells. pils2-1 (SALK_024808), pils2-2 (SALK_125391), pils5-1 (SALK_070653) and pils5-2 (SALK_072996) were obtained from the Nottingham Arabidopsis Stock Centre (NASC). Insertion sites were verified, homozygous lines selected and the decrease or absence of the respective PILS transcript was shown by RT-PCR. The pils2-2 and the pils5-2 mutants were crossed into DR5rev::GFP ${ }^{2}$. Gateway cloning was used to construct pPILS2::GFP::GUS, pPILS5::GFP::GUS, p35S::PILS1-7, p35S::GFP::PILS1-7, p35S::PILS1-7::GFP, pPILS5::PILS5::GFP and pMDC7_B(pUBQ)::PILS2. The PILS full-length genomic fragments, complementary DNA and promoter regions were amplified by PCR from genomic DNA and CDNA, respectively. The PCR was performed using the high fidelity DNA polymerase "I proof" (Bio-Rad). The primers used are given below. The full genomic and cDNA fragments were cloned into the pDONR221 (Invitrogen) vector and the promoter regions into pDONR$\mathrm{P} 4 \mathrm{P} 1$ using Invitrogen BP-clonase according to manufacturer's instructions. Coding sequences were transferred from the entry clones to gateway-compatible destination vectors (given below) using the Invitrogen LR clonase $(+)$ according to manufacturer's instructions. The resulting constructs were transformed into Col-0 plants by floral dipping in Agrobacterium tumefaciens liquid cultures. Yeast vectors were transformed into budding yeast (Saccharomyces cerevisiae) via electroporation. The p35S::PILS5::GFP line was crossed into pDR5rev::mRFP1er ${ }^{20}$. The following lines and constructs have been previously described: pDR5rev::mRFP1er ${ }^{20}$, pDR5rev::GFP $P^{2}$ and $p 35 s:: G F P:: H D E L^{21}$. Seeds were stratified at $4{ }^{\circ} \mathrm{C}$ for 2 days in the dark. Seedlings were grown vertically on half Murashige and Skoog medium. Plants were grown under long-day $\left(16 \mathrm{~h}\right.$ light $/ 8 \mathrm{~h}$ dark) conditions at $20-22{ }^{\circ} \mathrm{C}$.

Chemicals. 1-Naphthaleneacetic acid (NAA) was supplied by Duchefa, 2,4dichlorophenoxy acetic acid, oestradiol and propidium iodide by Sigma-Aldrich and ${ }^{3} \mathrm{H}$-indole-3-acetic acid $\left({ }^{3} \mathrm{H}\right.$-IAA), ${ }^{3} \mathrm{H}$-naphthalene-1-acetic acid $\left({ }^{3} \mathrm{H}-\mathrm{NAA}\right)$ and ${ }^{14} \mathrm{C}$-benzoic acid $\left({ }^{14} \mathrm{C}\right.$-BA) (specific radioactivity $\left.20 \mathrm{Ci} \mathrm{mmol}^{-1}\right)$ by American Radiolabelled Chemicals.

RNA extraction and quantitative real time PCR (qPCR). Whole RNA of seedlings was extracted using the RNeasy Mini Kit (Qiagen) in technical triplicates, the extracted RNA samples were treated with DNase (Ambion). qPCR analysis was performed using ICycler (Bio-Rad) with the Platinum SYBR Green qPCR SuperUDG kit (Invitrogen) following recommendations of the manufacturer. qPCR was carried out in 96-well optical reaction plates heated for $10 \mathrm{~min}$ to $95^{\circ} \mathrm{C}$ to activate hot-start Taq DNA polymerase, followed by 40 cycles of denaturation for $60 \mathrm{~s}$ at $95^{\circ} \mathrm{C}$ and annealing-extension for $60 \mathrm{~s}$ at $58^{\circ} \mathrm{C}$. Target quantifications were performed with specific primer pairs (given later) designed using Beacon Designer 4.0 (Premier Biosoft International). Expression levels were normalized to the expression levels of translation initiation factor EIF4A. The primers used are given later. Phenotype analysis. For analysis of the root length and lateral root density, plates were scanned on a flat-bed scanner. Root hairs were imaged with a binocular microscope (Leica). For hypocotyls analysis, seeds on plates were exposed to light for $3 \mathrm{~h}$ at $18^{\circ} \mathrm{C}$, and cultivated in the dark at $20^{\circ} \mathrm{C}$. Seedlings were imaged in real time with an infrared camera (Canon) to define the exact moment of germination and analysed 1, 3 and 5 days after germination. Hypocotyls, root and root hair lengths were measured with the ImageJ (http://rsb.info.nih.gov/ij/) software. Lateral root density for each seedling was obtained by calculating the number of lateral roots per root length unit 14 days after germination. For analysis of hypocotyls length, a minimum of 15 hypocotyls per condition or mutant line were analysed in each experiment. For analysis of root length and lateral root density, a minimum of 40 plants per condition or mutant line were analysed in each experiment. Means and standard errors were calculated and the statistical significance was evaluated by the student $t$-test using the GraphPad Prism5 (http://www.graphpad.com) software. For the analysis of root hair growth, 20 seedlings per transgenic line were imaged by binocular (Leica) and 20 root hairs (randomly chosen in the root hair zone) per seedling were measured with the ImageJ (http://rsb.info.nih.gov/ij/) software. The mean and standard error of the mean per transgenic line were calculated and the statistical significance was evaluated by the student $t$-test. To obtain the auxin-dependent root hair elongation, the same number of root hairs per seedling, seedlings per mutant line and condition were analysed as described above. The untreated mean average root hair length of the respective genotype was subtracted from the individual auxin-treated root hair length to obtain auxin induced growth in millimetre. The mean and standard error of the mean of the respective genotype were calculated and the statistical significance was evaluated by the student $t$-test using the GraphPad Prism5 (http://www.graphpad.com) software. All experiments were performed in at least three independent biological repetitions.

BY-2 plant material. Cells of tobacco line BY-2 (Nicotiana tabacum L., cv. Bright Yellow 2$)^{19}$ transformed with $p M D C 7 \_B(p U B Q):: P I L S 2$ were cultured in liquid cultivation medium $\left(3 \%(\mathrm{w} / \mathrm{v})\right.$ sucrose, $4.3 \mathrm{gl}^{-1}$ Murashige and Skoog salts, $100 \mathrm{mgl}^{-1}$ inositol, $1 \mathrm{mgl}^{-1}$ thiamine, $0.2 \mathrm{mgl}^{-1}$ 2,4-dichlorophenoxy acetic acid, and $200 \mathrm{mgl}^{-1} \mathrm{KH}_{2} \mathrm{PO}_{4}$ (pH 5.8). BY-2 cell lines were cultivated in darkness at $26^{\circ} \mathrm{C}$ on an orbital incubator (Sanyo Gallenkamp, Schöeller Instruments; 150 r.p.m., 32-mm orbit) and subcultured weekly. Stock BY-2 calli were maintained on media solidified with $0.6 \%(\mathrm{w} / \mathrm{v})$ agar and subcultured monthly.

Transient transformation of BY-2 cells and monitoring of cellular auxin signalling in BY2. Ten $\mathrm{ml}$ of three-day-old cells were harvested on filter paper by vacuum filtration and kept on plates with solid BY-2 medium. The cells were transformed via particle bombardment with a PDS 1000/He biolistic system (Bio-Rad) according to the manufacturer's instructions (http://www.bio-rad. com/webroot/web/pdf/lsr/literature/Bulletin_9075.pdf). $2 \mu \mathrm{l}$ of plasmid DNA $\left(0.05 \mu \mathrm{g} \mu \mathrm{l}\right.$ of the pDR5rev::mRFP1er contruct and $0.1 \mu \mathrm{g} \mu \mathrm{l}^{-1}$ of $p 35$ S::PILS2 and $p 35 S:: P I L S 5)$ was added to $6.25 \mu \mathrm{l}$ of $1.6-\mu \mathrm{m}$ diameter gold particles (dissolved in $50 \%$ glycerol). The suspension was supplemented with $2.5 \mu$ spermidine $(0.1 \mathrm{M}$ stock solution) and $6.25 \mu \mathrm{CaCl}_{2}$ (2.5 M stock solution). The particles were pelleted by centrifugation, washed twice with $70 \%$ and $100 \%$ ethanol and, subsequently, resuspended in $10 \mu \mathrm{l}$ of $100 \%$ ethanol. Cells were bombarded under a pressure of 1,100 pounds per square inch. The plates were sealed with Parafilm and kept in the dark for $18 \mathrm{~h}$ at $25^{\circ} \mathrm{C}$. For microscopic analysis, cells were gently transferred from the filter to a microscopy slide (in water) and subsequently covered with a cover slip. Samples were analysed via confocal microscopy. The pDR5rev::mRFPler expression was evaluated by defining the mean grey value (MGV) of each imaged cell (middle sections). For each experiment, confocal settings were defined based on the pDR5rev::mRFP1er signal of the control cells and remained unchanged during the respective experiments. Transformants were identified on the basis of the fluorescence of both proteins and imaged with a $\times 40$ objective. Every experiment/transformation was done in triplicate and for each condition a total number of at least 60 transformed cells were imaged. For each experiment, the means and standard errors were calculated and the statistical significance (independence between the two populations) was obtained by the student $t$-test using the GraphPad Prism5 (http://www.graphpad.com) software.

Immunocytochemistry. Whole-mount immunological staining on 5-day-old seedlings was done in an Intavis robot according to the described protocol ${ }^{22}$. The antibodies used at the final dilutions were monoclonal mouse anti-BIP2 (Hsc70) at 1:200 (Stressgen Bioreagents), monoclonal rabbit anti-GFP at 1:600 (Invitrogen). The secondary anti-mouse (Invitrogen) and anti-rabbit (SigmaAldrich) antibodies conjugated with $\mathrm{Cy} 3$ and Alexa488 respectively were used at 1:600 dilution.

Microscopy. Confocal microscopy was done with a Zeiss 710 microscope (Zeiss) or Leica SP5 (Leica). Fluorescence signals for GFP (excitation $488 \mathrm{~nm}$, emission peak $509 \mathrm{~nm}$ ), mRFP1 (excitation $561 \mathrm{~nm}$, emission peak $607 \mathrm{~nm}$ ) and propidium iodide (PI) staining (excitation $536 \mathrm{~nm}$, emission peak $617 \mathrm{~nm}$ ) were detected with a $\times 20, \times 40$ (water immersion) or $\times 63$ (water immersion) objective. Sequential scanning was used for double labelling to avoid crosstalk between channels. Fluorescence signal intensity was analysed with ImageJ (http://rsb.info.nih.gov/ij/) software and data were statistically evaluated with Microsoft Excel 2007.

Auxin transport assays in tobacco BY-2 cells, baker's yeast and Arabidopsis thaliana protoplasts. Auxin accumulation with 2-day-old BY-2 cells was measured as previously described ${ }^{14,23}$. The ${ }^{3} \mathrm{H}$-IAA was added to give a final concentration of $2 \mathrm{nM}$. Accumulation results were expressed as pmols of particular auxin accumulated per $10^{6}$ cells. The $0.5-\mathrm{ml}$ aliquots of cell suspension were collected continuously and accumulation of label was terminated by rapid filtration under reduced pressure on 22-mm-diameter cellulose filters. The cell cakes and filters were transferred to scintillation vials, extracted in $0.5 \mathrm{ml}$ of $96 \%$ ethanol for $30 \mathrm{~min}$, and afterwards $4 \mathrm{ml}$ of scintillation solution (EcoLite Liquid Scintilation Fluid, MP Biomedicals) was added. Radioactivity was determined by liquid scintillation counter Packard Tri-Carb 2900TR (Packard-Canberra, Meridian). Yeast ${ }^{3} \mathrm{H}$-IAA loading was quantified with the unspecific ${ }^{14} \mathrm{C}$-benzoic acid as control assayed in parallel and performed as previously described ${ }^{24}$. Relative export is calculated from yeast-retained radioactivity as follows: ((radioactivity in the yeast at time $t=10 \mathrm{~min}$ )-(radioactivity in the yeast at time $t=0)) \times(100 \%) /($ radioactivity in the yeast at $t=0 \mathrm{~min})$. Unspecific loading due to diffusion was eliminated by vector control subtraction. IAA export from Arabidopsis thaliana mesophyll protoplasts was analysed as described ${ }^{16}$.

HPLC metabolic profiling in tobacco BY-2 cells. Two-days-old BY-2 cells were prepared for the experiment by equilibration in uptake buffer as already described for accumulation assays ${ }^{14}$. Experiments were done in uptake buffer and under 
standard cultivation conditions. Cells were incubated with addition of $20 \mathrm{nM}^{3} \mathrm{H}$ IAA for a period of 0 and $20 \mathrm{~min}$. Cells and media (uptake buffer) were collected and frozen in liquid nitrogen ( $100 \mathrm{mg}$ of fresh weight and $5 \mathrm{ml}$ per sample). Extraction and purification of auxin metabolites in cells and media were performed as described ${ }^{25,26}$. The metabolites were separated on HPLC consisting of autosampler and 235C diode array detector (Perkin Elmer), column Luna C18 (2), $150 \times 4.6 \mathrm{~mm}, 3 \mu \mathrm{m}$ (Phenomenex, Torrance, USA), mobile phase A: $40 \mathrm{mM}$ $\mathrm{CH}_{3} \mathrm{COONH}_{4},(\mathrm{pH} 4.0)$ and mobile phase $\mathrm{B}: \mathrm{CH}_{3} \mathrm{CN} / \mathrm{CH}_{3} \mathrm{OH}, 1 / 1$, (v/v). Flow rate was $0.6 \mathrm{ml} \mathrm{min}{ }^{-1}$ with linear gradient $30-50 \% \mathrm{~B}$ for $10 \mathrm{~min}, 50-100 \% \mathrm{~B}$ for $1 \mathrm{~min}, 100 \%$ B for $2 \mathrm{~min}, 10-30 \%$ B for $1 \mathrm{~min}$. The column eluate was monitored on 235C DAD followed by Ramona 2000 flow-through radioactivity detector (Raytest $\mathrm{GmbH})$ after online mixing with three volumes $\left(1.8 \mathrm{ml} \mathrm{min}^{-1}\right)$ of liquid scintillation cocktail (Flo-Scint III, Perkin Elmer). The radioactive metabolites were identified on the basis of comparison of their retention times with authentic standards. For the results presentation the total integrated area of chromatogram plots has been normalized based on the equalization of total accumulated radiolabel.

In silico and phylogenetic analysis. PILS genes were identified via the SMARTprotein tool from EMBL (http://smart.embl-heidelberg.de/2) ${ }^{27,28}$. Phylogenetic tree of AtPILS was constructed with the DNA-man software version 4.0. PILS topologies were defined by the online HMM-top tool (http://www.enzim.hu/hmmtop/) ${ }^{29}$ and visualized by the TMRPres2D software (http://biophysics.biol.uoa.gr/TMRPres2D/ download.jsp $)^{30}$. PILS orthologues were identified with the online tool Plaza (http://bioinformatics.psb.ugent.be/plaza/) ${ }^{31}$.

Free IAA and conjugate measurements in Arabidopsis thaliana. For the quantification of free IAA and its amino acid conjugates, approximately $10 \mathrm{mg}$ of plant material was taken into analysis. The samples were processed as previously described $^{32}$ and quantified by UHPLC-MS/MS

Used primers and vectors. Genotyping primers: pils2-1 RP, CTGGAGAAACC TGACATCTCG; pils2-1 LP, GATTGAAGCCGGCTTAAATTC; pils2-2 RP, CTGGAGAAACCTGACATCTCG; pils2-2 LP, TACCATTGATCTGTCTTCG GG; pils5-1 RP, TTGAGACCCGTATCATTGGAG; pils5-1 LP, TGTCCTG ATAAAACCTTTTCAGG; pils5-2 RP, TACTGCACCGAAAATGAAACC; pils5-2 LP, TTGTACTATTTGCACCGGCTC.

Insert primer (LBb) (combine with RP): GCGTGGACCGCTTGCTGCAACT. RT-PCR primers used for insertion lines: PILS2 Fw, GCGATCATTATCGGATC AGT; PILS2 Rev, TTGCATACCTTGGACAGTAGTC; PILS5 Fw, TGTTGA AGCCCGTAATTCCATGAAC; PILS5 Rev, TTCATTGCGGACCCTTTAAT CAGC.

qPCR primers: PILS1 Fw, CGGTAACACAGCTCCACTTC; PILS1 Rev, GCAACAAGTAACGCACAACC; PILS2 Fw, GTGATGCTTGTACTTGGTGG TATG; PILS2 Rev, AACTTGAACATTGGATCTGCTGAG; PILS3 Fw, AGGCG ACCATGCAAGTGTTG; PILS3 Rev, GTGGTACAGCTAGATGACAGTGAG; PILS4 Fw, TGTCATAACTAAGCCTCCTTCAC; PILS4 Rev, CTCGCAACTCTC AGAATCTCC; PILS5 Fw, CTTGGAATAGTCTGTGTTCGGTAC; PILS5 Rev, GCACTGAGCATTCGTCTTGAG; PILS6 Fw, GCCTACATCAGTGCTCTCAG; PILS6 Rev, GCACTGAGCATTCGTCTTGAG; PILS7 Fw, TCCTCCAGACCCTC TCTTTCG; PILS7 Rev, ACAAGAAGATGACCGAGCACTC; EiF4a Fw, CTGGAGGTTTTGAGGCTGGTAT; EiF4a Rev, CCAAGGGTGAAAGCAAG AAGA.

Cloning primers: gDNA/cDNA: PILS1_Fw, GGGGACAAGTTTGTACAAAA AAGCAGGCTCGATGAGGATGAGGCTTTTGGATC; PILS1_Rev, GGGGAC CACTTTGTACAAGAAAGCTGGGTC(TCA)GGCTACGAGCCACATGAAG AATG; PILS2_Fw, GGGGACAAGTTTGTACAAAAAAGCAGGCTCGATGT CAGGTTTCTCCAGTGGAA; PILS2_Rev, GGGGACCACTTTGTACAAGAAA GCTGGGTC(TCA)TTGCATACCTTGGACAGTAGTCTC; PILS3_Fw, GGGG ACAAGTTTGTACAAAAAAGCAGGCTCGATGGTGAAGCTTTTGGAGCTG
TTC; PILS3_Rev, GGGGACCACTTTGTACAAGAAAGCTGGGTC(TCA)AGC TACAAGCCACATGAAGAATG; PILS4_Fw, GGGGACAAGTTTGTACAAAA AAGCAGGCTCGATGAAGCTTTTGGAGTTGTTCA; PILS4_Rev, GGGGAC CACTTTGTACAAGAAAGCTGGGTC(TCA)TGTCACAAGCCACATGAAGA ATG; PILS5_Fw, GGGGACAAGTTTGTACAAAAAAGCAGGCTCGATGGG ATTCTGGTCGTTGTTGGA; PILS5_Rev, GGGGACCACTTTGTACAAGAAA GCTGGGTC(TCA)GACTAACAAGTGAAGGAAGATGG; PILS6_Fw, GGGGA CAAGTTTGTACAAAAAAGCAGGCTCGATGATTGCTCGGATCCTTGCCG; PILS6_Rev, GGGGACCACTTTGTACAAGAAAGCTGGGTC(TCA)GAAGAG TATGTTAATGTAGAGTAC; PILS7_Fw, GGGGACAAGTTTGTACAAAAAA GCAGGCTCGATGGGTTTCTTAGAGTTGTTGGA; PILS7_Rev, GGGGACCA CTTTGTACAAGAAAGCTGGGTC(TCA)GGAGAGGATGGAGAGGAAGAT GG.

Promoter: PILS2_Fw, GGGGACAACTTTGTATAGAAAAGTTGCGAACTCC ATTGTTAACAGTAATAGC; PILS2_Rev, GGGGACTGCTTTTTTGTACAAA CTTGCCTCGATCTCACTATGTAAAGCTCG; PILS5_Fw, GGGGACAACTT TGTATAGAAAAGTTGCGCAATATACGTGACGTGGTCCACT; PILS5_Rev, GGGGACTGCTTTTTTGTACAAACTTGCCTTTTTATGTGGTTCTTTAGAC. Destination vectors: $p$ PILS::GFP/GUS: $p$ KGWFS7, p35S::PILS::GFP: $p H 7 F W G 2,0$, p35S::GFP::PILS: pH7WGF2,0, p35S::PILS::RFP: pK7RWG2,0, p35S::RFP::PILS: pK7WGR2,0 and p35S::PILS_D: pH7WG2D,1 (ref. 33), and pPILS::PILS::GFP: pK7m34GW,0 (ref. 34).

Oestradiol-inducible PILS: $p M D C 7 \_B(p U B Q)^{35}$ (p35S promoter was exchanged by the PUBQ10 promoter).

22. Sauer, M., Paciorek, T., Benkova, E. \& Friml, J. Immunocytochemical techniques for whole-mount in situ protein localization in plants. Nature Protocols 1, 98-103 (2006).

23. Delbarre, A., Muller, P., Imhoff, V. \& Guern, J. Comparison of mechanisms controlling uptake and accumulation of 2,4-dichlorophenoxy acetic acid, naphthalene-1-acetic acid, and indole-3-acetic acid in suspension-cultured tobacco cells. Planta 198, 532-541 (1996).

24. Bailly, A. et al. Modulation of P-glycoproteins by auxin transport inhibitors is mediated by interaction with immunophilins. J. Biol. Chem. 283, 21817-21826 (2008).

25. Dobrev, P. I., Havlíček, L., Vágner, M., Malbeck, J. \& Kamínek, M. Purification and determination of plant hormones auxin and abscisic acid using solid phase extraction and two-dimensional high performance liquid chromatography. J. Chromatogr. A 1075, 159-166 (2005).

26. Dobrev, P. I.\& Kamínek, M. Fast and efficient separation of cytokinins from auxin and abscisic acid and their purification using mixed-mode solid-phase extraction. J. Chromatogr. A 950, 21-29 (2002).

27. Schultz, J., Milpetz, F., Bork, P.\& Ponting, C. P. SMART, a simple modular architecture research tool: identification of signaling domains. Proc. Natl Acad. Sci. USA 95, 5857-5864 (1998).

28. Letunic, l., Doerks, T. \& Bork, P. SMART 6: recent updates and new developments. Nucleic Acids Res. 37, 229-232 (2009).

29. Tusnády, G. E. \& Simon, I. The HMMTOP transmembrane topology prediction server. Bioinformatics 17, 849-850 (2001).

30. Spyropoulos, I. C., Liakopoulos, T. D., Bagos, P. G. \& Hamodrakas, S. J. TMRPres2D: high quality visual representation of transmembrane protein models. Bioinformatics 20, 3258-3260 (2004).

31. Proost, S. et al. PLAZA: a comparative genomics resource to study gene and genome evolution in plants. Plant Cell 21, 3718-3731 (2009).

32. Pěnčík, A. et al. Isolation of novel indole-3-acetic acid conjugates by immunoaffinity extraction. Talanta 80, 651-655 (2009).

33. Karimi, M., De Meyer, B. \& Hilson, P. Modular cloning in plant cells. Trends Plant Sci. 10, 103-105 (2005).

34. Karimi, M., Inze, D. \& Depicker, A. GATEWAY vectors for Agrobacterium-mediated plant transformation. Trends Plant Sci. 7, 193-195 (2002)

35. Curtis, M. D. \& Grossniklaus, U. A gateway cloning vector set for high-throughput functional analysis of genes in planta. Plant Physiol. 133, 462-469 (2003) 